\title{
Diverses modalités de traitement des troubles d'apprentissage scolaire par thérapies visuelles: quelles sont les évidences scientifiques?
}

\author{
Amélie Ganivet, OD, M.Sc. \\ Isabelle Denault, OD \\ Rosanne Superstein, MD FRCSC \\ Nicole Fallaha, MD FRCSC
}

Clinique d'optométrie Granger Bernier et Associés 383 Boulevard du Séminaire Nord, suite 215

Saint-Jean-sur-Richelieu, Québec J3B 8C5

Hôpital Sainte Justine Département d'Ophtalmologie 3175 Chemin de la Côte-SainteCatherine Montréal, Québec

H3T 1C5
Résumé

Les troubles d'apprentissage sont des désordres complexes qui entravent le développement normal des processus d'acquisition et de traitement de l'information. Ils sont couramment rencontrés au sein de la population pédiatrique et peuvent se manifester sous diverses formes dont la plus fréquente est la dyslexie. Les troubles d'apprentissage ont une nature chronique et n'ont pas pour cause première des handicaps visuels, auditifs et/ ou moteurs, la déficience intellectuelle, la perturbation affective ni même un milieu défavorisé. Cependant, il est possible que ces troubles puissent coexister avec l'un ou l'autre de ces problèmes.

Puisque les enfants qui en sont atteints rencontrent souvent des difficultés de taille lors des apprentissages fondamentaux dès leur entrée à l'école primaire, il est essentiel de leur offrir un soutien adapté, un diagnostic précoce et une intervention efficace. Plusieurs hypothèses impliquant divers problèmes visuels ont été émises quant à la cause de la dyslexie et des troubles d'apprentissage. La présente revue de littérature traite de ces diverses hypothèses de traitement des troubles d'apprentissage scolaire par thérapies visuelles.

L'analyse démontre qu'il n'y a pas de preuves scientifiques suffisantes à ce jour prouvant que la thérapie visuelle, les lunettes d'entraînement, les exercices de poursuites et de saccades, les exercices perceptuels, les lunettes grossissantes, les filtres ou lentilles colorées ainsi que les prismes peuvent améliorer significativement les troubles d'apprentissage.

\section{Abstract}

Learning disabilities are complex disorders that interfere with the normal acquisition and processing of knowledge. In the pediatric population, dyslexia is the most common diagnosis. Learning disabilities are chronic in nature and are not caused by visual, auditory, motor, and intellectual difficulties or environmental factors. Those conditions may, however, coexist with the disorder.

Many of these problems present when children start elementary school, and it is important to offer early diagnosis and intervention. Various theories have evolved suggesting that visual troubles are the cause of dyslexia and learning disabilities. This article reviews the scientific basis of these theories and the evidence-based research. 
This review shows that there is not enough scientific evidence to support that vision therapy, training glasses, pursuit and saccade exercises, magnifying glasses, coloured lenses and/or overlays and prisms significantly help in coping with learning disabilities.

\section{MISE EN CONTEXTE}

Les troubles d'apprentissage sont des dysfonctionnements spécifiques et fréquents qui entravent le développement normal des processus d'acquisition. En 2006, selon Statistique Canada, 3,2\% des enfants Canadiens âgés entre 5 et 14 ans étaient affectés par un trouble d'apprentissage, ce qui représenterait environ un enfant par classe. ${ }^{[1]}$ Plusieurs hypothèses ont été émises en regard de l'étiologie des troubles d'apprentissage dans les dernières décennies. Comme ceux-ci ne sont pas encore parfaitement bien compris, une multitude de traitements non prouvés scientifiquement en sont dérivés. De ce fait, diverses thérapies visuelles sont parfois suggérées afin de traiter ces désordres. ${ }^{[2,3]}$ Par souci d'aider ces patients à centraliser leurs ressources et efforts afin d'atteindre leurs objectifs d'apprentissage, la recommandation de ces thérapies devrait se baser sur des données factuelles et scientifiques (« evidence-based »). ${ }^{[3,4]}$

Sous-tendant un caractère génétique, les troubles d'apprentissage sont chroniques et engendrés par une atteinte des fonctions neuropsychologiques. ${ }^{[5-7]}$ Ces désordres ne sont donc pas causés par une déficience intellectuelle, un déficit sensoriel, un mauvais encadrement scolaire ou un manque d'intérêt personnel. Les enfants qui en sont affectés possèdent généralement une capacité de raisonnement moyenne ou supérieure à la moyenne même s'ils éprouvent des difficultés à acquérir, comprendre, organiser et traiter les informations. ${ }^{[4,5,7]}$ Les principaux troubles d'apprentissage sont les suivants : la dyslexie, le trouble déficitaire de l'attention avec ou sans hyperactivité (TDA-H), la dysphasie, la dysorthographie, la dyspraxie, la dyscalculie et les troubles du spectre de l'autisme (TSA). La dyslexie est le désordre le plus fréquemment rencontré, affectant $80 \%$ des individus ayant des troubles d'apprentissage et jusqu'à 5-17\% de la population générale. ${ }^{[4-8]}$ Par conséquent, la dyslexie sera le trouble d'apprentissage principalement considéré dans le présent article.

Plusieurs hypothèses impliquant des atteintes visuelles ont été émises quant à la cause de la dyslexie et des troubles d'apprentissage. Bien que certaines divergences d'opinions persistent, l'hypothèse la plus largement acceptée à ce jour fait plutôt état d'un « déficit dans la composante phonologique du langage qui rend difficile l'utilisation du code alphabétique dans le décodage des mots écrits». ${ }^{[4]}$ Les troubles visuels parfois rencontrés seraient en fait attribuables à un manque d'expérience en lecture. ${ }^{[9-12]}$ Une étude récente démontre même qu'un entraînement en lecture basé sur la phonologie améliore non seulement les habiletés en lecture, mais également les fonctions visuelles. ${ }^{[9]}$ Ainsi, les anomalies visuelles parfois évoquées seraient une conséquence du trouble d'apprentissage et non la cause. ${ }^{[3,4,6,8-10,13]}$

Puisque la dyslexie est considérée comme un trouble phonologique du langage et non une dysfonction visuelle, des publications de l'Académie Américaine de Pédiatrie ainsi que de la Société Canadienne de Pédiatrie recommandent un traitement basé sur le décodage, la fluidité, le vocabulaire et la compréhension mais aussi plus spécifiquement sur la conscience phonémique et son application. ${ }^{[3-5,7,9,14,15]}$ De nature chronique, le trouble d'apprentissage ne disparaîtra pas avec l'âge. Toutefois, afin d'établir un plan d'intervention axé sur les besoins spécifiques de chaque enfant et de maximiser ses apprentissages, une prise en charge précoce s'appuyant sur la démarche scientifique est favorable. ${ }^{[7]}$

TRAITEMENT DES TROUBLES D’APPRENTISSAGE SCOLAIRE PAR THÉRAPIES VISUELLES : ÉTAT DES CONNAISSANCES

Certains problèmes de vision peuvent interférer avec le processus de lecture, créant ainsi des difficultés d'apprentissage réversibles suite à la correction du trouble visuel en cause. Par conséquent, il est primordial que tout enfant ayant un trouble ou des difficultés d'apprentissage ait un examen visuel complet dès les premières suspicions afin d'évaluer sa vision et sa santé oculaire. ${ }^{[3,6,16]}$ 
Néanmoins, il a été démontré que les enfants atteints de dyslexie ou de troubles d'apprentissage n'ont pas plus d'anomalies de leur fonction visuelle et de leur santé oculaire que les enfants non affectés par ces conditions. ${ }^{[4,6,8,14,17-19]}$ Leur apprentissage de matières telles que les mathématiques et le français peut être laborieux, mais ils réussissent souvent très bien dans d'autres sphères de développement nécessitant tout autant leurs aptitudes visuelles. Il n'y a actuellement pas de preuves scientifiques basées sur la médecine factuelle qui démontrent que de subtiles erreurs de réfraction ou de légers problèmes visuels pourraient provoquer ou augmenter la gravité des troubles d'apprentissage, diminuer l'efficacité visuelle ou encore amenuiser la réponse aux divers traitements éducationnels. ${ }^{[3,4,17,20]}$ L'hypothèse selon laquelle les enfants qui participent à une thérapie visuelle seraient conséquemment plus réceptifs aux divers programmes d'apprentissage n'est pas plus fondée. $[2,4,6,15,20-23]$

\section{L’ACUITÉ VISUELLE, LA RÉFRACTION ET LES LUNETTES D'ENTRAÎNEMENT}

Les enfants qui débutent l'apprentissage de la lecture et de l'écriture utilisent des textes dont les lettres sont de taille assez grande. Plus le niveau scolaire augmente, plus la taille des caractères diminue et plus la demande visuelle est accrue. Bien qu'une bonne vision soit importante, il n'est pas primordial d'avoir une résolution optimale afin de discerner les caractères utilisés au tout début des apprentissages scolaires. Il n'existe d'ailleurs aucune preuve démontrant que les lecteurs débutants atteints de myopie, d'hypermétropie et/ou d'astigmatisme léger à modéré ont plus de difficulté à apprendre à lire que les autres enfants. ${ }^{[4]}$ De petits degrés d'hypermétropie sont considérés normaux chez les jeunes enfants et n'ont généralement pas de signification pathologique. Ils constituent, au contraire, une étape du développement normal de l'œil. Les enfants ont des besoins visuels uniques basés sur leurs demandes visuelles et le développement de leur système optique. ${ }^{[24-28]}$ Les besoin réfractifs des enfants ne peuvent être extrapolés en fonction des besoins des adultes. Actuellement, il n'existe pas de règles absolues précisant les montants exacts des différentes amétropies requérant une correction optique ; les recommandations sont plutôt basées sur l'expérience clinique et différents consensus de professionnels de l'optométrie et ophtalmologie pédiatriques reconnus. ${ }^{[4,24-29]}$

Les enfants possèdent une capacité accommodative largement plus importante que les adultes. En effet, les enfants âgés entre 6 et 10 ans possèdent en moyenne 12,00 dioptries (D.) ou plus de fonction accommodative. ${ }^{[4,25,30]}$ Ils peuvent donc généralement compenser une hypermétropie modérée sans avoir de difficultés visuelles. La réfraction moyenne des enfants blancs aux États-Unis est d'environ 2,00 D. d'hypermétropie dans les 5 premières années de vie. ${ }^{[4]}$ Cette hypermétropie tend à diminuer progressivement durant l'adolescence. C'est pourquoi une hypermétropie légère à modérée n'a bien souvent pas besoin d'être corrigée, car elle a peu d'impact au niveau de la fonction oculo-visuelle. ${ }^{[24,25,27]}$

Il ne semble pas y avoir de probabilité accrue de dyslexie auprès des enfants ayant une hypermétropie non corrigée. En absence d'une diminution de l'acuité visuelle, il n’y aurait aucune corrélation entre les habiletés de lecture, les performances scolaires et le degré d'hypermétropie. ${ }^{[3]]}$ Des enfants de 6 ans n'ont généralement aucune réduction significative de l'acuité visuelle si l'hypermétropie n'excède pas 4,00 D. ; moins de $1 \%$ des enfants ont une hypermétropie plus forte. ${ }^{[25]}$ Une erreur de réfraction élevée de nature hypermétropique peut engendrer un inconfort visuel qui peut être considérable. Ainsi, ces enfants, se désintéressant des tâches requérant un effort visuel prolongé, pourraient développer des difficultés d'apprentissage consécutives. Cependant, celles-ci seraient réversibles suite à la correction du problème visuel. À l'inverse, un enfant ayant une hypermétropie élevée non corrigée et atteint de dyslexie ou d'un réel trouble d'apprentissage pourra remarquer une amélioration de son confort, de son acuité visuelle et même de ses performances scolaires suite à la correction de son hypermétropie, son trouble d'apprentissage étant possiblement amplifié par sa mauvaise vision. Toutefois, le trouble d'apprentissage va assurément persister en raison de sa nature plutôt neurologique que sensorielle.

Les enfants atteints de myopie non corrigée observent une diminution de leur acuité visuelle en vision éloignée et, par conséquent, peuvent avoir de la difficulté à voir le tableau en classe. Toutefois, ces enfants n'ont généralement pas de difficultés avec la vision de près surtout si la 
myopie est légère ou modérée. Malgré leur condition visuelle, il n’y aurait pas de corrélation entre la myopie faible et la réussite scolaire. ${ }^{[25]}$ La correction optique, si requise, doit être déterminée en tenant compte des besoins visuels de l'enfant en fonction de son âge. ${ }^{[25]}$ De plus, les études ont démontré que la sous correction autrefois utilisée pour diminuer la progression de la myopie serait sans fondement. ${ }^{[25]} \mathrm{Il}$ en est de même concernant l'hypothèse selon laquelle l'ajout d'un double-foyer pourrait diminuer la progression de la myopie. Cette hypothèse s'est avérée cliniquement non fondée. ${ }^{[4,20,25,32,33]}$

Chez les enfants d'âge scolaire, des degrés d'astigmatisme de moins de 1,50 D. ne produiraient qu'une dégradation minimale de la vision et ne causeraient pas d'amblyopie s'ils sont symétriques. ${ }^{[24-26]}$ Les astigmatismes obliques, toutefois, seraient plus dérangeants pour la vision. En général, il serait recommandé de corriger les astigmatismes de plus de 1,00 D. à 1,50 D. pour les enfants scolaires. Néanmoins, tout comme pour l'hypermétropie et l'astigmatisme, la décision de ne pas les corriger ne serait pas la cause d'un trouble d'apprentissage. ${ }^{[4,25]}$

L'amblyopie est caractérisée de façon fonctionnelle par une acuité visuelle réduite non améliorable par correction optique. Cette faiblesse de la vision engendre également une difficulté à distinguer les lettres à proximité l'une de l'autre. Chez les enfants atteints d'amblyopie bilatérale, il est possible que le niveau de lecture soit plus lent, mais ces enfants n'auront pas plus de dyslexie que les autres. ${ }^{[4]}$ De plus, les enfants atteints de nystagmus, de cataractes bilatérales ou d'anomalies de la santé oculaire peuvent avoir une diminution variable de leur acuité visuelle. ${ }^{[4]}$ Toutefois, les enfants souffrant d'un trouble de vision modéré à sévère sont aptes à apprendre à lire avec l'aide de corrections optiques adéquates et d'aides visuelles propres à la basse vision. Ainsi, en général, les maladies oculaires n'ont pas d'incidences sur l'habileté d'un enfant à apprendre à lire correctement.

En résumé, il n'existe aucune corrélation entre le rendement en lecture et de modestes anomalies de réfraction non corrigées. En se basant sur une médecine factuelle, il est non seulement inutile mais inadéquat de prescrire une faible correction dans le but de favoriser le traitement de la dyslexie et des troubles d'apprentissage. ${ }^{[4,6,20]}$ Toutefois, il est essentiel d'effectuer un examen visuel complet incluant un examen de l'état réfractif sous cycloplégie chez tous les enfants avec diagnostic ou suspicion d'un trouble d'apprentissage et ce, afin de détecter toute erreur de réfraction nécessitant une correction optique selon les standards généralement acceptés dans la profession.

\section{LES SACCADES ET LES FIXATIONS}

Les saccades sont de brefs et rapides mouvements oculaires entre deux zones servant à décoder l'environnement. Sollicitées lors de la lecture, elles peuvent être suivies d'une saccade de correction en cas d'imprécision par exemple lorsqu'un mot ou un groupe de mots a été incompris. Contrairement à certaines croyances, les mesures de saccades d'adultes témoins ou atteints de dyslexie ne sont pas différentes. ${ }^{[4,11,17,18,34-38]}$ Les saccades et fixations des enfants avec troubles d'apprentissage sont imprécises par rapport aux enfants d'un même âge, mais le sens du lien de causalité n'est pas clairement établi. Selon des études récentes, les saccades et les fixations des lecteurs dyslexiques sont semblables à celles de lecteurs témoins appariés plutôt en fonction d'un niveau similaire d'habileté en lecture que des attentes de leur âge. ${ }^{[9,13,39,40]}$

À ce jour, l'hypothèse du déficit phonologique est la plus largement acceptée pour expliquer la dyslexie. ${ }^{[3,4,6-9]}$ De ce fait, un entraînement basé sur la phonémique permettrait l'amélioration des fonctions visuelles en améliorant le niveau de lecture. Ainsi, les troubles visuels notés, attribuables à un manque d'expérience en lecture, seraient la conséquence et non la cause de la dyslexie. ${ }^{[9-12,40]}$ Les enfants atteints de dyslexie vont souvent perdre leur place en lisant, confondre certains sons, éprouver des difficultés à lire des mots plus rares ou élaborés. La lecture est lente, d'autant plus que le niveau demandé est supérieur aux capacités. Ces difficultés exigent un investissement d'énergie considérable en lecture et en compréhension et engendrent, par conséquent, des anomalies au niveau des poursuites et saccades. L'amélioration du niveau de lecture permettrait d'améliorer les saccades et les fixations, mais il n'y a pas de preuves scientifiques suffisantes qui confirment que des exercices de saccades et de poursuites visuelles 
pourraient aider le lecteur dyslexique à développer un meilleur niveau de lecture. $[2,4,10,23,35,40,41]$

Enfin, la majorité des individus atteints d'un trouble soit au niveau des motilités oculaires ou des mouvements oculaires ont une lecture et une compréhension normale. En effet, plusieurs enfants nés avec un strabisme important, un nystagmus ou encore avec une maladie oculaire affectant les mouvements oculaires excellent au niveau de leurs résultats académiques et de leur niveau de lecture. ${ }^{[3,23,42,43]}$ Ainsi, la dyslexie ne serait pas le résultat d'un déficit oculomoteur, mais plutôt le résultat d'un trouble au niveau central du traitement de l'information qui occasionne une difficulté au niveau du décodage et de la compréhension. Par conséquent, elle engendre des fixations plus longues et des saccades de corrections plus nombreuses lorsque le niveau de lecture demandé est supérieur aux capacités du lecteur.

\section{ACCOMMODATION}

L'accommodation est la capacité à se concentrer et à faire la mise à foyer au près. Elle assure la netteté des images selon différentes distances de vision. Les amplitudes accommodatives sont généralement maximales durant l'enfance jusqu'à l'âge de 10 ans, le pouvoir accommodatif diminuant ensuite naturellement avec l'âge. ${ }^{[44]}$ Il est donc rare d'observer une insuffisance accommodative chez un enfant. Un défaut accommodatif pourrait tout de même survenir chez un enfant avec une hypermétropie élevée non corrigée avec histoire d'infection virale, de trauma cérébral ou oculaire, de pathologie du tronc cérébral ou encore secondaire à la prise de médicament. ${ }^{[4]}$ L'hypothèse de la faiblesse accommodative d'enfants dyslexiques ou avec trouble d'apprentissage a amené plusieurs professionnels à promouvoir l'usage d'un double-foyer afin de la compenser. Bien que certaines études aient démontré une amplitude d'accommodation légèrement plus basse des enfants dyslexiques par rapport à des enfants normaux, l'amplitude des dyslexiques se situait dans les normes attendues pour leur âge. ${ }^{[8,45]}$ Ainsi, il n'y a pas de différence significative entre la capacité accommodative de patients ayant un trouble en lecture et celle de lecteurs de niveau normal. ${ }^{[8,13]}$ De plus, il n'y a pas de preuves scientifiques qu'une augmentation du grossissement augmente l'efficacité de la lecture. Une étude a même démontré que la correction de l'insuffisance accommodative n'avait pas d'impact significatif sur les mouvements oculaires et la fluidité lors de la lecture. ${ }^{[46]}$ Par conséquent, toute thérapie visant à diminuer l'effort accommodatif d'un enfant ayant des troubles d'apprentissage prétextant que son trouble en est la cause est non fondée scientifiquement. ${ }^{[2,4,6,20]}$

\section{VISION BINOCULAIRE}

L'équilibre oculomoteur parfait est rare dans la population pédiatrique et générale. La plupart des individus présentent une faible ésophorie ou exophorie asymptomatique qui sont considérées dans les limites normales. ${ }^{[4,17,19]}$ Plusieurs études ont investigué la fonction binoculaire et l'accommodation d'enfants avec troubles d'apprentissage scolaire et dyslexie. Aucune relation de causalité n'a pu être mise en évidence. ${ }^{[17,19,47]}$

L'insuffisance de convergence représente une difficulté à fusionner correctement et efficacement un objet situé à une distance rapprochée. Lorsqu'un effort de convergence est difficile à surmonter, il peut provoquer divers symptômes d'inconfort visuel tels que fatigue visuelle, maux de tête, vision floue en lecture, diplopie en vision rapprochée, difficulté à se concentrer pendant des périodes prolongées de travail au près. De plus, certains facteurs tels que le manque de sommeil, la maladie, une fatigue générale peuvent aggraver le problème. ${ }^{[4]}$ La prévalence de l'insuffisance de convergence serait d'environ $3 \%$ à $5 \%$ de la population. ${ }^{[4]}$ Toutefois, en raison de la différence entre les critères de diagnostic, certaines études rapportent des données qui peuvent différer. Autant une difficulté accommodative qu'une insuffisance de convergence peuvent interférer avec le confort de la lecture. ${ }^{[3,8,45]}$ Ces troubles visuels doivent être traités s'ils sont reconnus comme problématiques selon les critères énoncés pour la population générale. De ce fait, le traitement de l'insuffisance de convergence peut aider au niveau du confort de la lecture et des travaux en vision rapprochée en permettant une lecture prolongée plus aisée. ${ }^{[20,48]}$ Également, si les difficultés de lecture chez un individu sont secondaires à une anomalie de l'accommodation ou de la convergence, ces difficultés disparaîtront une fois le trouble visuel traité. ${ }^{[4]}$ Toutefois, puisque ces troubles visuels ne sont pas la cause de la dyslexie, leur entraînement n'aura pas d'impact sur les capacités de décodage 
et de compréhension en lecture. ${ }^{[4]}$

Tout comme la majorité des enfants, ceux ayant des troubles d'apprentissage aiment jouer à des jeux vidéo. L’utilisation de jeux vidéo requiert une bonne coordination œil-main, de la concentration pendant une période prolongée, une accommodation efficace, une convergence active ainsi qu'une bonne perception visuelle. Par conséquent, si ces déficits étaient la cause majeure des troubles de lecture, ces enfants rejetteraient cette tâche qui nécessite également une utilisation intensive de leurs capacités visuelles. ${ }^{[4,6]}$

\section{LES LENTILLES ET FILTRES COLORÉS}

L'utilisation de lentilles ou de filtres colorés visant à améliorer le confort et les performances en lecture chez les individus ayant des troubles d'apprentissage est très controversée. Certains sont d'avis que l'utilisation de lentilles ou filtres jaunes permettrait d'améliorer le contrôle de l'attention visuelle et des mouvements oculaires chez certains enfants par stimulation cérébrale. L'utilisation de lentilles ou filtres bleus, quant à elle, améliorerait la concentration et conséquemment, la lecture. ${ }^{[49,50]}$ D'autres avancent que le contrôle de l'accommodation et de la convergence serait influencé par une sensibilité à certaines longueurs d'ondes de la lumière, créant un stress visuel lors de la lecture. L'utilisation de certains filtres colorés correspondant à ces différentes longueurs d'onde permettrait une réduction de ce stress et une lecture plus efficace. ${ }^{[4,51-53]}$ Toutefois, plusieurs études ont statué que le choix approprié de la couleur du filtre bénéfique pour chaque individu serait inconsistant et non répétable. ${ }^{[6,54-59]}$ D'autres études ont démontré que l'utilisation de lentilles ou filtres colorés n'avait aucun effet bénéfique sur la fonction visuelle et les performances en lecture. ${ }^{[55,60]}$ Comme aucun consensus ne peut être établi à ce jour, l'utilisation de filtres colorés afin de traiter les enfants avec troubles d'apprentissage n'est pas cliniquement justifiée pour le moment. ${ }^{[4,6,14,54,56-59,61-63]}$

\section{LES PRISMES}

L'utilisation de prismes chez des patients avec troubles d'apprentissage a été rapportée. Certains affirment que des prismes base en haut pourraient être utilisés pour traiter l'exophorie ou l'insuffisance de convergence tandis que des prismes base en bas seraient employés dans le traitement de l'ésophorie ou l'excès de convergence. ${ }^{[20]}$ Les prismes base en bas seraient parfois même utilisés afin de faciliter l'adaptation des individus pour qui une faible correction d'hypermétropie serait prescrite. ${ }^{[20]}$

D'autres prétendent qu'une pleine correction de toute hétérophorie permettrait de diminuer la fatigue visuelle lors de la lecture. Selon cette hypothèse, de petites hétérophories occasionneraient un effort de compensation afin de maintenir une vision binoculaire adéquate: elles devraient donc être corrigées complètement à l'aide du prisme approprié. ${ }^{[64,65]}$ Plusieurs réfutent toutefois cette hypothèse et ne recommandent pas l'utilisation de prismes car il n'améliore pas les performances en lecture. ${ }^{[6,667]}$ Les effets bénéfiques rapportés pourraient, selon eux, être attribués à un effet placebo. ${ }^{[66,67]}$ À ce jour, aucune preuve scientifique encourageant l'utilisation des prismes dans le but d'améliorer la lecture des enfants avec troubles d'apprentissage n'a été clairement établie. $[4,6,20,21,57,66,67]$

\section{DISCUSSION}

La dyslexie serait une difficulté d'origine neurobiologique à décoder et à comprendre un langage écrit. Elle correspond à un déficit dans le processus de la structure du son du langage écrit ou parlé (défaut phonologique). ${ }^{[6]} \mathrm{Il}$ s'agit d'un désordre persistant et chronique dont les causes sont multifactorielles et sous influence génétique. ${ }^{[6]}$

Les atteintes oculo-visuelles peuvent interférer avec le processus d'apprentissage de la lecture mais elles ne sont pas la cause de la dyslexie ni des troubles d'apprentissage. En effet, bien que la vision soit fondamentale pour lire, le cerveau doit analyser les images visuelles transmises. Statistiquement, les enfants ayant de la dyslexie ou des troubles d'apprentissage associés ont le même niveau de fonction visuelle et le même niveau de santé oculaire que les enfants sans ces conditions. ${ }^{[3,8,18,19]} \mathrm{Il}$ n'y a pas de preuves scientifiques à ce jour démontrant que la thérapie visuelle, les lunettes d'entraînement, les exercices de poursuites et de saccades, 
les exercices perceptuels, les lunettes grossissantes, les filtres ou les lentilles colorées ainsi que les prismes peuvent significativement améliorer les performances de l'enfant ayant des troubles d'apprentissage. ${ }^{[2,4,6,7,14,20-22,57,68]}$ Ces approches peuvent donner de faux espoirs aux parents et autres intervenants et de ce fait, possiblement retarder une intervention ayant un meilleur potentiel bénéfique pour l'enfant. De plus, les coûts engendrés par ces thérapies sont substantiels et le temps requis pour les effectuer non négligeable. Les études statuant sur l'amélioration des apprentissages via ces thérapies sont en fait des études non contrôlées scientifiquement ou encore basées sur des cas anecdotiques. ${ }^{[2,4]}$ Les bénéfices avancés seraient plutôt secondaires aux autres traitements éducationnels traditionnels souvent effectués de façon combinée avec ces thérapies visuelles et/ou à l'effet placebo de tels procédés.

\section{CONCLUSION}

La détection précoce des enfants ayant des troubles d'apprentissage et leur référence vers les professionnels appropriés sont essentielles afin de fournir le support nécessaire à ces enfants et leur famille. Il est primordial que ces enfants aient un examen visuel complet avec cycloplégie afin de s'assurer qu'ils ne présentent pas d'atteinte visuelle entravant leur fonction visuelle et pouvant ainsi amplifier les symptômes de dyslexie/troubles d'apprentissage. Une approche multidisciplinaire est privilégiée chez ces enfants. En plus de l'évaluation de la vision, une évaluation de la santé, du développement, de l'audition et si nécessaire une intervention médicale et/ou psychologique devra être effectuée. ${ }^{[4,6,13,14,20,21]}$ En terminant, la dyslexie et les troubles d'apprentissage sont des problèmes complexes n'ayant malheureusement pas de solutions simples. Toutefois, il est recommandé que toutes les thérapies proposées soient scientifiquement justifiées afin de créer des attentes réalistes et de favoriser le développement de l'enfant.

\section{BIBLIOGRAPHIE}

1. Statistique_Canada. 2006; http://www.statcan.gc.ca/ daily-quotidien/071203/dq071203a-fra.htm.

2. Rawstron JA, Burley CD, ad Elder MJ. A systematic review of the applicability and efficacy of eye exercises. J Pediatr Ophthalmol Strabismus 2005;42(2):82-8.

3. Granet DB. Learning disabilities, dyslexia, and vision: the role of the pediatric ophthalmologist. J AAPOS 2011;15(2):119-20.

4. Handler SM, et al. <AU: Please provide two more author names. $>$ Learning disabilities, dyslexia, and vision. Pediatrics 2011;127(3):e818-56.

5. Gabrieli JD. Dyslexia: a new synergy between education and cognitive neuroscience. Science 2009;325(5938):280-3.

6. American Academy of Ophthalmology. Policy statement - learning disabilities, dyxlexia and vision. 2009; http://www.aao.org/about/policy/upload/ Learning-Disabilities-Dyslexia-Vision-2009.pdf. Accessed May 1, 2013.

7. Siegel LS. Perspectives on dyslexia. Paediatr Child Health 2006;11(9):581-7.

8. Wahlberg-Ramsay M, et al. <AU: Please provide two more author names.>Evaluation of aspects of binocular vision in children with dyslexia. Strabismus 2012;20(4):139-44.

9. Olulade OA, Napoliello EM, Eden GF. Abnormal visual motion processing is not a cause of dyslexia. Neuron 2013;79(1):180-90.

10. Medland C, Walter H, Woodhouse JM. Eye movements and poor reading: does the developmental eye movement test measure cause or effect? Ophthalmic Physiol Opt 2010;30(6):740-7.

11. Olson RK, Kliegl R, Davidson BJ. Dyslexic and normal readers' eye movements. J Exp Psychol Hum Percept Perform 1983;9(5):816-25.

12. Hutzler F, et al. Perhaps correlational but not causal: no effect of dyslexic readers' magnocellular system on their eye movements during reading. Neuropsychologia 2006;44(4):637-48.

13. Olitsky SE, Nelson LB. Reading disorders in children. Pediatr Clin North Am 2003;50(1):213-24.

14. Committee on Children with Disabilities, American
Academy of Pediatrics (AAP) and American Academy of Ophthalmology (AAO), American Association for Pediatric Ophthalmology and Strabismus (AAPOS). Learning disabilities, dyslexia, and vision: a subject review. Pediatrics 1998; 102(5):1217-9.

15. A-C Bernard-Bonnin, S.c.d.p., Le recours à la médecine parallèle dans le traitement des enfants atteints de trouble de déficit de l'attention avec hyperactivité. Paediatr Child Health 2002;7(10):721--30.

16. Koller HP. An ophthalmologist's approach to visual processing/learning differences. J Pediatr Ophthalmol Strabismus 2002;39(3):133-42; quiz 169-70.

17. Hall PS, Wick BC. The relationship between ocular functions and reading achievement. J Pediatr Ophthalmol Strabismus 1991; 28(1):17-9.

18. Polatajko HJ. Visual-ocular control of normal and learning-disabled children. Dev Med Child Neurol 1987;29(4):477-85.

19. Palomo-Alvarez C, Puell MC. Binocular function in school children with reading difficulties. Graefes Arch Clin Exp Ophthalmol 2010;248(6):885-92.

20. Barrett BT. A critical evaluation of the evidence supporting the practice of behavioural vision therapy. Ophthalmic Physiol Opt 2009;29(1):4-25.

21. Kavale K, Mattson PD. "One jumped off the balance beam": meta-analysis of perceptual-motor training. J Learn Disabil 1983;16(3):165-73.

22. Burke MJ. Dyslexia and vision therapy. http://www. drmilesburke.com/eye-conditions/dyslexia-andvision-therapy.html.

23. American Academy of Ophthalmology. Complementary therapy assessment: vision therapy for learning disabilities. 2001; www.one.aao.org/ complementary-therapy-assessmentsĐ/visiontherapy-learning-disabilities. Accessed May 1, 2013.

24. Miller JM, Harvey EM. Spectacle prescribing recommendations of AAPOS members. J Pediatr Ophthalmol Strabismus 1998;35(1):51-2.

25. Donahue SP. Prescribing spectacles in children: a pediatric ophthalmologist's approach. Optom Vis Sci 2007;84(2):110-4.

26. American Academy of Ophthalmology. Preferred practice pattern. 2012; http://one.aao.org/preferred- 
practice-pattern/amblyopia-ppp--september-2012. Accessed May 1, 2013.

27. Leat SJ, et al. Prescribing for hyperopia in childhood and teenage by academic optometrists. Optom Vis Sci 2011;88(11):1333-42.

28. Donahue SP, et al. Guidelines for automated preschool vision screening: a 10-year, evidence-based update. J AAPOS 2013;17(1):4-8.

29. Leat SJ. To prescribe or not to prescribe? Guidelines for spectacle prescribing in infants and children. Clin Exp Optom 2011;94(6):514-27.

30. Sterner B, Gellerstedt M, Sjostrom A. The amplitude of accommodation in 6-10-year-old children - not as good as expected! Ophthalmic Physiol Opt 2004;24(3):246-51.

31. Helveston EM, et al. Visual function and academic performance. Am J Ophthalmol 1985;99(3):346-55

32. Gwiazda J, et al. A randomized clinical trial of progressive addition lenses versus single vision lenses on the progression of myopia in children. Invest Ophthalmol Vis Sci 2003;44(4):1492-500.

33. Gwiazda JE, et al. Accommodation and related risk factors associated with myopia progression and their interaction with treatment in COMET children. Invest Ophthalmol Vis Sci 2004;45(7):2143-51.

34. Black JL, et al. A detailed study of sequential saccadic eye movements for normal- and poor-reading children. Percept Mot Skills 1984;59(2): 423-34.

35. Brown B, et al. Tracking eye movements are normal in dyslexic children. Am J Optom Physiol Opt 1983;60(5):376-83

36. Brown B, et al. Predictive eye movements do not discriminate between dyslexic and control children. Neuropsychologia 1983;21(2):121-8.

37. Black JL, et al. Smooth pursuit eye movements in normal and dyslexic children. Percept Mot Skills 1984;59(1):91-100.

38. Biscaldi M, Fischer B, Aiple F. Saccadic eye movements of dyslexic and normal reading children. Perception 1994;23(1):45-64.

39. Black JL, et al. Dyslexia: saccadic eye movements. Percept Mot Skills 1984;58(3): 903-10.

40. Bucci MP, et al. Immaturity of the oculomotor saccade and vergence interaction in dyslexic children: evidence from a reading and visual search study. PLoS One 2012;7(3):e33458.

41. Metzger RL, Werner DB. Use of visual training for reading disabilities: a review. Pediatrics 1984;73(6):824-9.

42. MacDonald JT, et al. Reading skills in children and adults with albinism: the role of visual impairment. J Pediatr Ophthalmol Strabismus 2012;49(3):184-8.

43. Hodgetts DJ, et al. Normal reading despite limited eye movements. J AAPOS 1998;2(3):182-3.

44. Grosvenor T. Primary Care optometry, anomalies of refraction and binocular vision. 3rd ed. Oxford, UK: Butterworth-Heinemann, 1996:665.

45. Palomo-Alvarez C, Puell MC. Accommodative function in school children with reading difficulties. Graefes Arch Clin Exp Ophthalmo 2008;246(12):1769-74

46. Abdi S, et al. The influence of accommodative insufficiency on reading. Clin Exp Optom, 2007;90(1):36-43.

47. Evans BJ, Drasdo N, Richards IL. Dyslexia: the link with visual deficits. Ophthalmic Physiol Opt 1996;16(1):3-10.

48. Rouse MW, et al. Frequency of convergence insufficiency in optometry clinic settings. Convergence Insufficiency and Reading Study (CIRS)
Group. Optom Vis Sci 1998;75(2):88-96.

49. Hall R, et al. A comparison of two-coloured filter systems for treating visual reading difficulties. Disabil Rehabil 2013;35(26):2221-6.

50. Dyslexia Research Trust: Vision and coloured filters. ; www.dyslexic.org.uk/research-visual.html. Accessed July 8, 2014.

51. Harris D, MacRow-Hill SJ. Application of ChromaGen haploscopic lenses to patients with dyslexia: a doublemasked, placebo-controlled trial. J Am Optom Assoc 1999;70(10):629-40.

52. Harris D. Lenses for colour vision deficiency and reading difficulties in optometric practice. 2009 http://www.optometry.co.uk/uploads/articles/ April\%2010\%2009\%20Clinical.pdf. Accessed July 8, 2014.

53. Bouldoukian J, Wilkins AJ, Evans BJ. Randomised controlled trial of the effect of coloured overlays on the rate of reading of people with specific learning difficulties. Ophthalmic Physiol Opt 2002;22(1):55-60.

54. Ritchie SJ, Della Sala S, McInthsoh RD. Irlen colored filters in the classroom: a 1-year follow-up. Mind Brain Educ 2012;6:74-80.

55. Menacker SJ, et al. Do tinted lenses improve the reading performance of dyslexic children? A cohort study. Arch Ophthalmol 1993;111(2):213-8.

56. Woerz M, Maples WC. Test-retest reliability of colored filter testing. J Learn Disabil 1997;30(2):214-21.

57. Shainberg MJ. Vision therapy and orthoptics. Am Orthopt J 2010;60:28-32.

58. Iovino I, et al. Colored overlays for visual perceptual deficits in children with reading disability and attention deficit/hyperactivity disorder: are they differentially effective? J Clin Exp Neuropsychol 1998;20(6):791-806

59. Ritchie SJ, Della Sala S, McIntosh RD. Irlen colored overlays do not alleviate reading difficulties. Pediatrics 2011;128(4):e932-8.

60. Palomo-Alvarez C, Puell MC. Effects of wearing yellow spectacles on visual skills, reading speed, and visual symptoms in children with reading difficulties. Graefes Arch Clin Exp Ophthalmol 2013;251(3):945-

61. Evans BJ, Drasdo N. Tinted lenses and related therapies for learning disabilities - a review. Ophthalmic Physiol Opt 1991;11(3):206-17.

62. Wilkins AJ, Sihra N, Myers A. Increasing reading speed by using colours: issues concerning reliability and specificity, and their theoretical and practical implications. Perception 2005;34(1):109-20.

63. Gole GA., et al. Tinted lenses and dyslexics--a controlled study. SPELD (S.A.) Tinted Lenses Study Group. Aust N Z J Ophthalmol 1989;17(2):137-41.

64. Pestalozzi D. Ophthalmologic aspects of dyslexia: the influence of full prismatic correction of heterophoria on dyslexic symptoms. Ann N Y Acad Sci 1993;682:397-9.

65. Pestalozzi D. [Further observations of dyslexia patients with prism correction]. Klin Monbl Augenheilkd 1992;200(5):614-9.

66. Dysli M, Vogel N, Abegg M. Reading performance is not affected by a prism induced increase of horizontal and vertical vergence demand. Front Hum Neurosci 2014;8:431

67. Kommerell G, Kromeier M. [Prism correction in heterophoria]. Ophthalmologie 2002;99(1):3-9.

68. Helveston EM. Visual training: current status in ophthalmology. Am J Ophthalmol 2005;140(5):903-10. 